\title{
61st Annual Prairie Urological Association: Abstracts
}

\author{
Cite as: Can Urol Assoc J 2014;8(3-4):S1-7. http://dx.doi.org/10.5489/cuaj.1981
}

\section{Factors predicting surgical intervention in pediatric nephrolithiasis patients \\ Esther Jun (student), Todd Alexander, Peter Metcalfe}

Division of Urology, University of Alberta, Edmonton, AB

Objective: The prevalence of kidney stones in children is increasing. Consequently, the need to intervene surgically is also. However, clear indications for surgical intervention in pediatric nephrolithiasis are lacking. As a result, management of stones is currently based on patient preference and subjective assessment of whether it is feasible surgically to perform stone removal. We therefore set out to identify patient and stone characteristics that predict the necessity for surgery.

Methods: A retrospective analysis of 63 pediatric renal stone patients presenting to the University of Alberta Hospital from 1990 to 2013 was performed. These 63 patients presented with a total of 143 stones. Univariate and multivariate analysis are being conducted to assess for patient and stone characteristics requiring surgical intervention.

Results: Thus far, we have found that presentation with multiple bilateral stones is more common in individuals requiring surgery. Furthermore, the presence of a high creatinine and hypocitrituria were associated with surgical intervention. Conversely, surgery was less often performed in patients presenting with hypercalcemia and increased $1,25(\mathrm{OH})_{2} \mathrm{D}_{3}$. Stone characteristics associated with surgical intervention included large stone size, growth in stone size over time, movement in location, being present in the ureter and being composed of calcium oxalate. Conversely, small stones, composed of calcium phosphate, were more likely to pass without surgical intervention.

Conclusion: Our study provides some individual and stone characteristics associated with surgical intervention. Hopefully, these observations can contribute to the establishment of guidelines for when a stone should be monitored and allowed to pass, as opposed to when a stone should be surgically removed before it requires emergency care.

Source of Funding: Northern Alberta Urology Foundation

\section{Pediatric pyeloplasties: A comparison of symptomatic and screened patients}

Mark Assmus (student), Darcie Kiddoo, Peter Metcalfe

Division of Urology, University of Alberta, Edmonton, $A B$

Objective: Over the last 25 years, expectant management of antenatal hydronephrosis has become the standard of care, with very few asymptomatic, antenatally detected UPJ obstructions undergoing surgery. However, despite ubiquitous screening, many patients develop symptoms from their UPJ and require surgery. We hypothesize that a significant number of pyeloplasties are performed in symptomatic patients who had normal antenatal imaging.

Methods: We performed a retrospective chart review examining the management of 68 pediatric patients who underwent 72 pyeloplasties at the Stollery Children's Hospital in Edmonton from 2007 to 2013. Data collection included the presence of antenatal hydronephrosis, imaging results, as well as indications and outcomes of surgery.

Results: $60 \%(43 / 72)$ of pyeloplasties had symptomatic indications for surgery, while $40 \%(29 / 72)$ had imaging indications. Of the 43 symptomatic cases, $37 \%(16 / 43)$ were actively being followed for persistent hydronephrosis, and $12 \%$ (5/43) were previously followed, but their hydronephrosis was felt to have resolved. Regardless of the indication, $80 \%$ had imaging improvement at 1-year post-surgery. 65 patients had pre-surgical MAG3 scans with ipsilateral function of $41 \%$ (range $10 \%-50 \%$ ). $37 \%(10 / 27)$ of these displayed $\mathrm{a} \geq 10 \%$ decrease in function prior to surgery. Post-surgically, $40 \%(4 / 10)$ of these patients returned to within $90 \%$ of their initial differential function.

Conclusion: Despite ubiquitous access to antenatal screening, excellent sensitivity for diagnosing hydronephrosis, and active postnatal surveillance, the majority of pyeloplasties at our institution had symptomatic indications. This reinforces our belief that current screening strategies are unable to accurately define which patients will benefit from surveillance and asymptomatic surgery.

Source of Funding: Northern Alberta Urology Foundation

Previously Presented: 3rd Annual University of Alberta Research Day, October 2013

\section{Multicystic dysplastic kidney in children: Is long-term radiographic follow-up necessary?}

Vitor da Silva, Michael Leonard, Luis Guerra

Children's Hospital of Eastern Ontario, Ottawa, ON

Objective: Multicystic dysplastic kidney (MCDK) is relatively common, usually diagnosed by antenatal ultrasound in 1 in 4300 births. The majority of cases carry low morbidity and good prognosis. Despite this, longterm follow-up is commonplace, due to concerns of possible malignant transformation and development of hypertension. We set out to define the need for long-term follow-up, and consolidate the recommendations of the current literature given the relatively low rate of associated comorbidities in this cohort of patients.

Methods: An IRB approved retrospective analysis of patients with MCDK followed at our institution between 1990 and 2013 was carried out. Data were analyzed regarding length of follow-up, rate of MCDK involution, malignant transformation, hypertension and renal failure.

Results: Records of 201 patients were reviewed. Mean length of follow-up was 7.1 years (SD 5.1). Males were affected at a ratio of $1.3: 1$, and the right side was affected at a ratio of 0.9:1. Overall, 93\% (183/196) of patients were diagnosed by prenatal ultrasound. Vesicoureteric reflux was found in $19 \%(23 / 120)$ of patients who had a VCUG. The MCDK kidney underwent complete involution in 38\% (76/201) of cases over the course of follow-up. There were no cases of malignant transformation. The incidence of hypertension and renal failure were 5\% (10/201) and 3\% (6/201) respectively. Conclusion: MCDK carries a low rate of associated comorbidity. Based on this small series, long-term urological follow-up is not necessary in patients with a normal contralateral kidney. After confirmation of contralateral renal growth, compensatory hypertrophy and adequate renal function, followup should be done by the primary care physician in the form of regular blood pressure checks and urinalysis. A larger series would be necessary to validate these findings.

Source of Funding: This project is not funded. 


\section{Intravesical Onabotulinumtoxin A use in open pediatric urologic reconstruction cases effect on perioperative com- plications and hospital stay in children with neurogenic bladder}

Carolina Fermin, Heena Singh, Anthony Cook, William Hyndman, Bryce Weber

Alberta Children's Hospital, Calgary, $A B$

Objective: With the advent of oral anticholinergic medications and intravesical Onabotulinumtoxin A (BTA) over the past decade, the indications for lower tract reconstruction have diminished. However, there remain a number of patients who require reconstruction for continence and upper tract preservation. However, in children requiring operative intervention, the use of anticholinergics postoperative can increase the risk of serious complications such as ileus, constipation, and prolonged hospital stay. We have hypothesized that intraoperative intravesical BTA could significantly decrease the potential risk of postoperative bowel complications due to anticholinergic medication.

Methods: From January 2011 to December 2013 all patients at Alberta Children's Hospital undergoing lower tract reconstruction for neurogenic bladder were retrospectively reviewed. 21 patients were identified who either underwent appendicovesicostomy (13), bladder augmentation (2), or either bladder neck procedure (1) or combination thereof (5). These patients were divided into two groups, those who underwent intravesical BTA (10 units/kg of BTA was administered maximum dose 200 units) at the time of the reconstruction versus those who did not.

Results: None of the patients, who received intraoperative BTA injection $(0 / 13)$, required postoperative anticholinergic medications for treatment of bladder spasms compared to $5 / 8$ in the control group $(p=0.04)$. Postoperative ileus was documented in $3 / 13$ in botulinum toxin-A group verses $6 / 8$ in the control group $(p=0.11)$, one patient in control group required operative intervention for a bowel obstruction. The mean hospital stay was 5.6 days in BTA group vs. 8.1 days in the control group.

Conclusion: Intravesical BTA during lower urinary tract reconstruction in children with neurogenic bladder is an effective strategy to decrease the need for postoperative anticholinergic medication and potentially decrease postoperative ileus and decrease hospital stay.

Source of Funding: This project is not funded.

\section{H NMR serum metabolomics analysis may distinguish between stages of renal cell carcinoma}

Oluyemi S. Falegan, Rustem A. Shaykhutdinov, Mark W. Ball, Mohamad E. Allaf, Hans J. Vogel, Eric M. Hyndman

University of Calgary, Calgary, AB; Johns Hopkins Medical Institution, Baltimore, MD

Objective: To determine if $1 \mathrm{H}$ NMR based serum metabolomics is able to differentiate between benign and malignant renal masses.

Methods: Patients with renal lesions identified by CT scan had their serum collected after fasting before surgery. Serum from 40 patients were identified to have malignant lesions and 13 patients with benign lesions were assessed with $1 \mathrm{H}$ NMR based metabolomics. The groups were stratified by their pathology and 1H NMR serum metabolomics analysis was performed to study the changes in the metabolic signature of renal cell carcinoma (RCC). These profiles were then used to predict benign from malignant lesions.

Results: Using the Chenomx software package, 55 metabolites could be detected in each of the 53 serum samples (40 RCC and 13 controls) that were analyzed. Using multivariate statistical analysis, alterations in the levels of ornithine, creatine and methanol among other metabolites was detected in RCC relative to controls. Orthogonal partial least square discriminate analysis (OPLS-DA) plots discriminated between controls and pT1 $(\mathrm{R} 2=0.608, \mathrm{Q} 2=0.451)$ and pT3 $(\mathrm{R} 2=0.619, \mathrm{Q} 2=0.441)$ disease groups.

Conclusion: The results from this small preliminary study show that serum metabolomics combined with multivariate statistical analysis may be useful in differentiating between benign and metastatic RCC cases. This has significant potential clinical implications suggesting that metabolomics could serve as a non-invasive and economical alternative for renal biopsies. Source of Funding: University of Calgary

\section{Inhibition of pyruvate dehydrogenase kinase increases apoptosis and reduces proliferation and angiogenesis in renal cell carcinoma}

Adam Kinnaird, Gopinath Sutendra, Roxane Paulin, Peter Dromparis, Evangelos Michelakis

Department of Medicine, Division of Urology, University of Alberta, Edmonton, $\mathrm{AB}$

Objective: Solid tumours, such as renal cell carcinoma (RCC) exhibit a distinct metabolic phenotype known as the Warburg Effect. This principle describes cancer's preferential usage of cytoplasmic glycolysis (Gly) over mitochondrial glucose oxidation (GO) even in normoxia. This usage of Gly provides several growth advantages to cancer cells including increased angiogenesis and decreased apoptosis. The pyruvate dehydrogenase kinase (PDK) inhibiting small molecule dichloroacetate (DCA) decreases mitochondrial membrane potential $(\Delta \psi \mathrm{m})$ and increases mitochondrial reactive oxygen species (mROS) in cancer cells thereby inducing apoptosis, inhibiting angiogenesis and decreasing tumor growth in-vitro and in-vivo. The objective of this project is to assess the potential therapeutic benefit of the novel agent DCA on RCC by evaluating changes in proliferation, apoptosis and angiogenesis.

Methods: Two human kidney cell lines were used: (1) A proximal tubule (PT) epithelial cell line and (2) a clear cell RCC line (786-0). Cells were treated with 0.5 and $5 \mathrm{mM}$ DCA. Mitochondrial $\Delta \psi \mathrm{m}$ and mROS were assessed using live cell imaging with TMRM and mitosox, respectively. Tumor proliferation and apoptosis were measured using the markers ki67 and TUNEL, respectively. Nuclear localization of P53 was detected by immunofluoresence. PDH activity and $\hat{I}_{ \pm}$-ketoglutarate were measured using commercially available kits and phosphorylation by immunoblotting. Angiogenesis was assessed in-vitro by matrigel assay.

Results: RCC cells have more hyperpolarized $\Delta \psi \mathrm{m}(\mathrm{TMRM}, \mathrm{p}<0.001)$ and less mROS (Mitosox, $\mathrm{p}<0.001$ ) than PT cells. Treatment with DCA reversed these changes in the RCC line without significantly altering PT $\Delta \psi \mathrm{m}$ or $\mathrm{mROS}$. This is associated with a dose-responsive increase in PDH activity $(p<0.05)$ and increased levels of the Krebs cycle metabolite $\hat{I} \pm-K G$ $(p<0.01)$. RCC cells treated with DCA demonstrate increased nuclear localization of P53 $(p<0.01)$, which is associated with decreased markers of proliferation $(p<0.001)$, increased rates of apoptosis $(p<0.001)$, and a decreased rate of growth in vitro. Supernatant, containing the angiogenic signals from RCC cells was placed on microvascular endothelial cells. The supernatant from RCC cells increased vascularity (tubular structures and total length), which was blocked by DCA treatment $(p<0.05)$.

Conclusion: DCA is a novel, inexpensive, oral chemotherapeutic agent that reverses the mitochondrial remodeling of RCC, thereby inhibiting the Warburg effect. Treatment with DCA decreases proliferation and angiogenesis while increasing apoptosis in a human RCC lines. Animal xenograft experiments are currently underway.

Source of Funding: CIHR and AIHS Clinical Investigator Program

\section{Building an innovative delivery model for overactive blad-} der (OAB): A multidisciplinary approach

Daniel Yanko, Trafford Crump, Richard Baverstock, Kevin Carlson vesia [Alberta Bladder Centre], Department of Surgery, Division of Urology, University of Calgary, Calgary, AB

Objective: Successful treatment of OAB is dependent on accurate diagnosis, patient education, behavioural modifications, physical therapies, and the ability to offer appropriate medical and surgical interventions when appropriate. This can be difficult to provide in a conventional urological setting. In 2011, vesia [Alberta Bladder Centre] was developed as a multidisciplinary clinic for lower urinary tract disorders, including OAB. The new delivery system aims to ensure: 1 ) timely access, 2) reduce the cost on the health care system, and 3) reduce unnecessary diagnostics. The purpose of this study is to describe and report on a new model for delivering $\mathrm{OAB}$ care. 
Methods: To assess the effect of the vesia clinic on OAB care, a retrospective analysis of the electronic record was undertaken. A comparison of one full year of $O A B$ visits before and after the implementation of the vesia $\mathrm{OAB}$ model was performed.

Results: The year prior to the vesia delivery model being implemented, the clinic saw 150 unique patients for 173 OAB-related visits (average frequency of visits per patient $=1.15$ ). On average, these patients cost the healthcare system $\$ 142.25$ (all costs in 2012 dollars). Ninety-one of $(60 \%)$ these patients underwent a cystoscopy. The year after the vesia delivery model was implemented, the clinic saw 1,035 unique patients for 1,776 OAB-related visits (average frequency of visits per patient = 1.72). On average, these patients cost the healthcare system $\$ 112.67$. Three hundred thirteen (30\%) of these patients underwent cystoscopy. Overall, the vesia model increased OAB consults by nearly 900 unique patients, increased the frequency of visits by $49 \%$ while decreasing the average patient cost by $21 \%$.

Conclusion: The growing demand for $\mathrm{OAB}$ care necessitated the development of an innovative delivery model. Based on our analysis, the vesia $\mathrm{OAB}$ model represents an approach capable of increasing access for $\mathrm{OAB}$ care, while reducing overall costs to the healthcare system.

Source of Funding: CUA and Pfizer Incontinence Fellowship

\section{Stemming the tide of mild to moderate post-prostatectomy incontinence: $A$ retrospective comparison of transobtur- ator male slings and the artificial urinary sphincter Nathan Hoy, Keith Rourke}

Division of Urology, University of Alberta, Edmonton, $A B$

Objective: Urinary incontinence is a devastating post-prostatectomy complication. Prior to the advent of male slings, mild-moderate postprostatectomy incontinence (PPI) was typically managed with an artificial urinary sphincter (AUS). Although the AUS remains the gold standard, most patients with mild-moderate PPI prefer a sling without evidence of procedural equivalence. This study compares outcomes of the two procedures for the management of mild-moderate PPI.

Methods: A single centre retrospective review of 124 patients (76 transobturator sling, $48 \mathrm{AUS}$ ) with mild-moderate PPI requiring intervention over an 8-year period. Primary outcomes included continence and change in number of incontinence pads. Secondary outcomes included global patient satisfaction, improvement, and complication rates. Mild to moderate incontinence was defined as requiring $<5$ incontinence pads per day. Results: There was no significant difference in age at time of continence procedure (66.2 vs. 68.1 years; $p=0.17)$, or prostate cancer characteristics (PSA, Gleason score, number of cores positive on biopsy, pathologic $T$ score) for slings and AUS respectively. Patients receiving an AUS were significantly older at time of prostatectomy (62.2 vs. 64.7 years; $p=0.05)$, had a higher Charlson comorbidity index score (3.1 vs. $3.9 ; \mathrm{p}=0.0007$ ), and more likely to have previous radiotherapy (3.9 vs. $14.6 \%$; $\mathrm{p}=0.045$ ). Robotic prostatectomy ( 40.8 vs. $8.3 \%$; $p<0.001)$ was more common in the transobturator sling group. Median length of follow up was 7 months for the sling group and 11 months for the AUS group. There was no difference in overall continence rates, $88.2 \%$ vs. $87.5 \%(p=0.79)$, rate of improvement, $94.7 \%$ vs. $95.8 \%(p=1.00)$, or satisfaction, $93.4 \%$ vs. $91.7 \%(p=0.73)$, for slings and AUS, respectively. Complication rates were equivalent $(19.7 \%$ vs. $16.7 \%$; $p=1.00)$, though there was a significantly higher proportion of Clavien-Dindo Grade 3 complications with AUS $(0 \%$ vs. $75 \%$; $p=0.006)$, including device infection, or erosion requiring explantation. Postoperative transient urinary retention was significantly higher with slings at $18.7 \%$ vs. $5.7 \%(p=0.011)$.

Conclusion: For mild to moderate PPI there is no difference with respect to continence, satisfaction, or improvement rates, between AUS and slings. Complications arising with AUS tend to be more severe and require operative intervention more frequently. Our study supports the use of male slings as first line treatment for mild-moderate PPI.

Source of Funding: This project is not funded.

Previously Presented: 3rd Annual University of Alberta Research Day, October 2013

\section{Urethral transection does not influence erectile function after anterior urethroplasty: A prospective analysis}

Jeff Zorn, Keith Rourke

Division of Urology, University of Alberta, Edmonton, $\mathrm{AB}$

Objective: Urethral reconstruction is an effective therapy for urethral stricture of diverse etiologies. Urethroplasty, however involves surgical dissection of neurovascular structures that may provide erectile potency, thus posing is a theoretical risk of erectile dysfunction. Stricture location and transection of the urethra are possible risk factors for erectile dysfunction after anterior urethroplasty. The purpose of this study is to prospectively evaluate erectile function post-urethral reconstruction and to examine the effect urethral transection on potency.

Methods: Patients undergoing anterior urethral reconstruction completed an International Index of Erectile Function (IIEF) questionnaire at baseline and postoperatively at 6 months follow-up to assess potency. The Erectile Function (EF) domain ( 6 questions; 30 points possible) of the IIEF was evaluated pre and post-reconstruction in a prospective manner with clinically significant decline in EF defined as a 5-point decrease in EF domain score. Overall mean change in EF domain score from baseline was compared using the paired t-test and the proportions of patients with adverse change in $\mathrm{EF}$ were compared using Fishers exact analysis. Results: A total of 46 anterior urethral reconstructions with complete baseline and 6 month postoperative IIEF scores were included in the study. The average age was 47 years with a mean stricture length of $3.4 \mathrm{~cm}$. Thirty-nine of the 46 reconstructions were for strictures entirely in the bulbar urethra with the remaining 7 involving the penile urethra. Twentytwo of the reconstructions involved complete transection of the urethra while the others had various non-transecting techniques. Mean baseline and postoperative EF domain scores were 16.85 and 17.17 respectively $(p=0.82)$. Overall, at a minimum of 6 months follow-up the incidence of adverse change in EF (5-point decrease in EF domain score) was $15.2 \%$ (7). Urethral transection did not significantly affect the occurrence of erectile dysfunction $(\mathrm{p}=0.69)$. Stricture location (bulbar versus penile) was also not found to have a significant impact on EF postoperatively $(p=0.29)$. Conclusion: Urethroplasty can result in a decline in erectile function in some patients but overall is associated with minimal adverse change in erectile function. Urethral transection does not affect the occurrence of erectile dysfunction 6 months after urethroplasty.

Source of Funding: Northern Alberta Urology Foundation

\section{The effect of preoperative urine culture status on urethro- plasty complications and outcomes}

Keith Rourke, Callum Lavoie, Adam Kinnaird

Division of Úrology, University of Alberta, Edmonton, AB

Objective: Urethroplasty remains the most definitive treatment of urethral stricture. Many patients with urethral stricture experience urinary tract infection or have chronic bacteruria. It is unclear if colonization of the urinary tract affects urethroplasty outcomes. The objective of this study is to evaluate the effect of a positive preoperative urine culture on urethroplasty complications and outcomes.

Methods: A retrospective review of 734 urethroplasties performed by a single surgeon was performed. All patients received preoperative culture sensitive parenteral antibiotics (typically an aminoglycoside and a first generation cephalosporin). Positive urine culture was defined as $>108$ bacteria per $\mathrm{ml}$ or in select instances $>106$ of specific pathogenic bacteria (Pseudomonas, etc.). The effect of a positive urine culture was investigated by examining differences in postoperative wound infection, UTI, and stricture recurrence. Statistical analysis was performed using a Fisher's 2-sided exact test.

Results: Overall, the rates of wound infection, UTI and stricture recurrence were $4.7 \%, 4.0 \%$ and $8.7 \%$ respectively. Comparison between the positive urine culture group and the negative urine culture group revealed a statistically significant difference in the average age of the patients $(p=0.0001)$, but otherwise no statistically significant differences in average stricture length $(p=0.9781)$, revision urethroplasty $(p=0.05231)$ or average co-morbidity score $(p=0.1086)$. Between the positive and negative urine culture groups, the rate of postoperative wound infection were not 
statistically significant $(p=0.5587)$. Likewise, the rates of postoperative UTI $(p=1.000)$ and stricture recurrence $(p=0.2965)$ were not found to be significantly different with a mean follow-up of 50 months. The specific type of pathogen did not alter the rate of complications.

Conclusion: The presence of a positive urine culture with administration of clinically appropriate antimicrobials does not appear to affect urethroplasty complications or outcomes.

Source of Funding: Northern Alberta Urology Foundation

Previously Presented: Northwest Urological Society

\section{The devastated urethra: our experience in the manage- ment of refractory post-prostate therapy urethral strictures} Daniel Yanko, Kevin Carlson, Trafford Crump, Bryce Weber, Richard Baverstock

vesia [Alberta Bladder Centre], Department of Surgery, Division of Urology, University of Calgary, Calgary, $\mathrm{AB}$

Objective: Urethral strictures may develop from the treatment of both benign and malignant prostate etiologies and can be challenging to manage. We reviewed our local experience in managing refractory postprostate therapy urethral strictures (PPUS) via a novel treatment pathway. Methods: We performed a retrospective analysis of all patients at our Centre diagnosed with a urethral stricture following various prostate therapies between May 2008 and October 2013. Multiple variables were assessed, including: initial diagnosis, method of stricture treatment and number of treatments. All patients underwent initial endoscopic management followed by a standardized self-catheterization program developed at our Centre.

Results: Forty-six patients were identified with initial diagnoses of prostate cancer in $28(61 \%)$ and benign prostatic hyperplasia $(\mathrm{BPH})$ in 18 $(39 \%)$. All patients underwent initial endoscopic management [direct visual internal urethrotomy (DVIU) or transurethral incision of bladder neck (TUIBN)] by us (38) and/or the referring urologist (8). In total, a mean of $1.50(95 \% \mathrm{Cl} 1.22-1.78)$ endoscopic procedures were performed per patient. $41(89 \%)$ patients were stabilized following endoscopic resection combined with a standardized self-dilation program. Five (11\%) patients were refractory to the above measures and required more invasive treatment: $2(4.3 \%)$ cystectomy with ileal conduit, $2(4.3 \%)$ open vesicourethral anastamotic revision, $1(2.2 \%)$ YV-plasty. All three patients who underwent open bladder neck reconstructive procedures remain with stable outlets at cystoscopic follow-up (6-48 months).

Conclusion: In our large cohort of refractory PPUS, we have demonstrated successful outcome in the majority of cases with minimal operative intervention. In select cases, a variety of reconstructive surgical procedures may be required. These observations support our novel simplified approach to these refractory patients.

Source of Funding: This project is not funded.

\section{Observations, outcomes and complications of urethro- plasty for bulbomembranous stenosis after radiation treat- ment for prostate cancer}

Keith Rourke

Division of Urology, University of Alberta, Edmonton, $A B$

Objective: Urethral stenosis is an under-reported complication of radiotherapy for prostate cancer. The objective of this study is to report outcomes and complications of urethral reconstruction for radiation induced bulbomembranous urethral stenoses.

Methods: A retrospective review of 735 urethral reconstructions performed by a single surgeon from August 2004 to May 2013 identified 35 patients requiring reconstruction for radiation induced bulbomembranous stenosis with a minimum 6 months of follow-up. Primary outcome was urethral patency (cystoscopy) and secondary outcomes were 90-day complications, de-novo incontinence, erectile dysfunction and LUTS. Outcomes were compared between reconstructive techniques using Fisher's exact test.

Results: Of the 35 patients 20 and 15 had stenosis related to external beam radiation therapy (EBRT) or brachytherapy (BT) respectively. Mean stricture length was $3.5 \mathrm{~cm}$ with 40.7 months of follow-up. Many (42.9\%) patients presented with 'permanent' suprapubic catheters and $45.7 \%$ reported preoperative erectile dysfunction. Reconstruction was performed by excision and primary anastomosis (epa) in $23(65.7 \%)$ patients while 12 required tissue transfer with either buccal mucosa graft $7(20.0 \%)$ or penile island flap $5(14.3 \%)$. Thirty patients $(85.7 \%)$ achieved cystoscopic patency with no difference between techniques $(p=0.317)$. A 90-day complication rate of $28.2 \%$ was observed (all Clavien 1-2) with no difference between techniques $(p=1.000)$. Adverse change in incontinence occurred in $25.7 \%$ of patients but was $13.3 \%$ when excluding those who had a previous TURP. Postoperatively $40 \%$ of patients described persisting storage LUTS and $23.3 \%$ described adverse change in erectile function (exclusively in the epa group).

Conclusion: Reconstruction of radiation induced bulbomembranous strictures yields satisfying patency rates. However, radiation induced urethral stenosis is not an isolated problem as many patients suffer from persisting storage LUTS, erectile dysfunction or incontinence as a consequence of treatment either before or after urethroplasty.

Source of Funding: This project is not funded.

\section{Intravesical OnabotulinumtoxinA for bladder dysfunction in a real world clinical practice}

Ted Hoyda, Heena Singh, Daniel Yanko, Bryce Weber, Kevin Carlson, Richard Baverstock

vesia [Alberta Bladder Centre], Department of Surgery, Division of Urology, University of Calgary, Calgary, $\mathrm{AB}$

Objective: Intravesical BOTOX (OnabotulinumtoxinA, BTA) injections for lower urinary tract dysfunction was first described in 1987, and has been used at our institution since 2004. It was approved for use in North America for refractory neurogenic detrusor overactivity (NDO) in 2012, and for idiopathic detrusor overactivity (IDO) in Canada in October 2013. BTA remains off-label for refractory bladder pain syndrome (BPS). Few studies have been published to confirm the long-term safety and efficacy of intravesical BTA, nor the adherence to this form of treatment. We report our long-term 'real-world' clinical experience with BTA for refractory bladder storage dysfunction (NDO, IDO, BPS) in our high-volume institution, with attention to baseline data, growth in uptake, and persistence with therapy.

Methods: A retrospective chart review of all patients injected between July 20, 2009 (when charts became available via EMR) and October 30, 2013 was performed.

Results: Overall a total of 1256 injections were performed on 433 unique patients with mean age of 57 years (range: 19-91). The majority of patients $(79 \%)$ were injected under local anesthesia only. The number of BTA treatments ranged from 1 to 14 . Sixty-four percent of patients have received more than 1 injection while $41 \%$ have received 3 or more, and $14 \% 6$ or more. Overall, $36 \%$ discontinued BTA at any time due to attrition, lack of effect, or change in bladder management. In 2010, 210 injections (18/ month) were performed, while 262 were done in 2011 (22/month), and 330 in 2012 (28/month). In the first 10 months of 2013, 370 injections have been performed (31/month). Indications included NDO in $51 \%$, IDO in $40 \%$ and BPS in $6 \%$. For the NDO group, $60 \%$ were female, compared to $80 \%$ of the IDO and $96 \%$ of the BPS groups. In the NDO group, $28 \%$ had spinal cord injury (SCI), 34\% multiple sclerosis (MS), and $38 \%$ had other causes. Over half (55\%) of all BTA recipients stopped or never used anticholinergic medication, $34 \%$ stayed on anticholinergics and $11 \%$ were restarted on an anticholinergic or Mirabegron. Ten percent of the entire cohort initiated clean intermittent catheter $(\mathrm{CIC}), 26 \%$ already performed $\mathrm{CIC}$, and $17 \%$ had indwelling catheters or suprapubic tubes. No serious adverse events or hospital admissions were observed. Conclusion: Intravesical BTA is a well-tolerated and safe procedure that is performed under local anesthetic in the majority of cases. Repeat treatments are common and popularity of BTA for patients with bladder dysfunction is growing. A significant number of patients initiated $\mathrm{CIC}$ in our blended cohort. Persistence with treatment is high, at 57\%, despite the challenging nature of the patient population.

Source of Funding: This project is not funded. 
A single centre contemporary analysis of the utility of retrograde urethrogram in the management of pelvic fracture urethral injuries

Conrad Maciejewski, Keith Rourke

Division of Urology, University of Alberta, Edmonton, $\mathrm{AB}$

Objective: Initial management of pelvic fracture urethral injury (PFUI) calls for assessment with retrograde urethrogram (RUG) then either urethral realignment or placement of a suprapubic catheter with delayed reconstruction. We aim to assess the utility of RUG for the management of these injuries and examine management strategies employed at our Level 1 Trauma Centre.

Methods: The Alberta Trauma Registry is a comprehensive database of all major traumas at the University of Alberta Hospital. The database from 2001-2013 was queried for all traumas with an Injury Severity Score (ISS) $>12$, injury code for pelvic fracture, and text containing "bladder" or "urethra." Follow up was cystoscopy and retrograde urethrogram. Failure was defined as patients developing stenosis requiring surgical intervention.

Results: 26 patients were identified with pelvic fracture urethral injuries. Mean age was $36 \pm 13$ years and mean ISS score was $26.7 \pm 7.6 .77 \%$ (20/26) of patients had RUG performed at initial assessment. 21 patients had complete disruption, and 5 had partial disruption. 12 patients were managed with suprapubic catheter, and 14 with placement of an aligning catheter with no significant difference in ISS between the two groups. Only 2 patients had successful unassisted catheter insertion. Of the 6 patients who did not receive RUG, 5 (83\%) had complete disruption and required operative insertion suprapubic catheter insertion. Urethral stenosis occurred in 6 realignment cases (43\%), and 11 (92\%) with suprapubic catheter $(\mathrm{p}<0.05)$.

Conclusion: Patients sustaining PFUI have a significant severity score, but this does not typically influence urologic management. Nearly all patients without RUG had high-stage injuries, were very unlikely to have blind aligning catheter placement and typically require suprapubic catheter insertion. RUG does not significantly alter management, and definitive diagnosis and management of PFUI occurs at time of endoscopy.

Source of Funding: This project is not funded.

A contemporary analysis of predictors of lymph node positivity at radical prostatectomy and clinical outcomes at a tertiary care referral centre

Kamilojot Kaler, Tad Kroczak, Premal Patel, Rebekah Rittberg, Jasmir Nayak, Jeffery Saranchuk, Darrel Drachenberg

Department of Urology, University of Manitoba, Winnipeg, MB

Objective: The extent of pelvic lymph node dissection (PLND) at the time of radical retropubic prostatectomy (RRP) varies among surgeons and institutions. Differences mainly focus on candidacy for node dissection and the limit of dissection (limited vs. extended). We analyzed our local experience of lymph node outcomes.

Methods: In this retrospective study with institutional board ethics, data was collected on consecutive patients undergoing RRP with PLND from January 2003 to June 2013 by two academic uro-oncologists. Extracted information included surgical pathology and patient, biopsy, and biochemical recurrence (BCR) characteristics. A univariate and multivariate analysis was conducted using SAS software.

Results: 420 consecutive patients, of whom 411 underwent a RRP with PLND and 9 aborted prostatectomies, had median follow-up of 46 months. Median patient age was 60 with a mean preoperative PSA of 11. Overall lymph node metastases rate was $16.1 \%$. Of these $\mathrm{N} 1$ patients, the average number of positive nodes was 2.3 with an average removal of 13.4 nodes. Anatomic regions of positive pelvic lymph node metastasis were 54 percent in hypogastric area, 13 percent in obturator area, and 33 percent were external iliac. 2 patients with $\mathrm{N} 1$ disease died and 79 percent had BCR. Median time from treatment to BCR was 4.5 months with a median PSA of 0.28 . Multivariate analysis showed that year of surgery $(p=0.04)$, Gleason $4+3(p=0.0003)$, and the biopsy ratio of total cancer length: total length of all cores $(p<0.0001)$ was associated with node positivity.
Conclusion: This contemporary series of RRP for prostate cancer shows a high rate of lymph node metastases (16\%). Performing a limited pelvic lymph node dissection would under stage $54 \%$ of our patients. To our knowledge, this study is the first to demonstrate that the ratio of preoperative biopsy total cancer length: total length of all cores was associated with node positivity.

Source of Funding: This project is not funded.

Previously Presented: University of Manitoba Urology Research Day

Natural history of a large active surveillance cohort of prostate cancer patients with 5-year median follow-up: Predictors of undergoing treatment and surgical outcomes in a realworld setting

Kamilojot Kaler, Premal Patel, Tad Kroczak, Rebekah Rittberg, Jasmir Nayak, Ross MacMahon, Jeffery Saranchuk, Darrel Drachenberg Department of Urology, University of Manitoba, Winnipeg, MB

Objective: Active Surveillance (AS) is an alternative to radical treatment for patients diagnosed with low risk prostate cancer (PCa). These patients are monitored with the intent to treat if the cancer progresses or by patient preference. We assessed our centre's experience with AS.

Methods: In this retrospective study with institutional board ethics, patients with an active diagnosis of PCa with Gleason $3+4$, T2b, and PSA $<20$ and patients who chose AS were included. Data analyzed included patient, biopsy, and surgical pathology characteristics, and disease outcomes. Multivariate analysis was utilized to identify predictors of undergoing radical treatment on AS using SAS software.

Results: Over a median follow up of 5.6 years, 289 patients were on AS at our centre with $114(39 \%)$ patients ultimately requiring treatment. Of the treated patients the median age was 65 with each patient requiring a median of 2 biopsies. Median interval to first follow-up biopsy was 12 months, and 20 months between all subsequent biopsies. $71 \%$ of patients had Gleason 3+3 when starting on active surveillance. In our multivariate model Gleason upgrade on surveillance biopsy $(p<0.0001)$, PSA change ( $p=0.0125)$, and absolute change in cancer length among all cores $(p<0.0001)$ was predictive of failing active surveillance. PSA doubling time approached significance $(\mathrm{p}=0.0535)$. Age, year of diagnosis, and PSA density were not predictive of receiving treatment. 23\% of patients were found to have Gleason upgrade on final surgical pathology. In addition, of the 114 treated patients $30 \%$ developed biochemical recurrence. No patient died of PCa in our study period.

Conclusion: In our study period, $39 \%$ of patients went on to treatment from AS. Total cancer linear distance change, PSA change, and Gleason upgrade were predictive of treatment. In terms of surgical pathology, twenty three percent were upgraded. No patient died of PCa.

Source of Funding: This project is not funded.

Previously Presented: University of Manitoba Urology Research Day

Utility of preoperative 3 Tesla pelvic phased-array magnetic resonance imaging in prediction of extracapsular extension of prostate cancer and its impact on surgical margin status: Experience at a Canadian tertiary academic health science centre

Ian Wright, Justin Lee, Jen Hoogenes, Colm Boylan, Bobby Shayegan

McMaster University, Hamilton, ON

Objective: Magnetic resonance imaging (MRI) of the prostate is rapidly gaining ground in preoperative assessment and surgical planning of patients undergoing radical prostatectomy (RP). However, published reports of its predictive ability to detect extracapsular extension (ECE) have varied significantly in the literature, regardless of the type of MR imaging employed. The specific MRI technology and the experience of the radiologist play key roles in the predictive ability of the imaging modality. We sought to evaluate the ability of 3 Tesla (3T) pelvic phased-array MRI to predict ECE prior to RP and the subsequent effect on surgical margin status in a cohort of patients treated by a single surgeon.

Methods: We retrospectively evaluated 48 preoperative RP patients who 
underwent 3T pelvic phased-array MRI at the discretion of the surgeon, based on the clinical probability of adverse pathological features. All MRIs were read by an expert genitourinary radiologist prior to RP. Tumor stage based on MRI was compared to pathological stage, and specificity, sensitivity, positive predictive value (PPV), and negative predictive value (NPV) of MRI in predicting ECE were calculated. Positive surgical margin rates of patients with a finding of ECE on MRI were compared to those without ECE on MRI.

Results: Forty-two (88\%) patients were of intermediate or high risk based on D'Amico criteria. The MRI reports predicted $19(40 \%)$ patients to be positive for ECE, while final pathology revealed that 9 of these patients were positive for ECE, with 2 having positive surgical margins. Of the $29(60 \%)$ patients who were not predicted to have ECE based on MRI, 14 actually had positive ECE on pathology, with 12 of these patients having positive surgical margins. Preoperative 3T pelvic phase-array MRI achieved a sensitivity of $44 \%$ and a specificity of $61 \%$ in predicting ECE on surgical pathology. The PPV and NPV were $55 \%$ and $50 \%$, respectively. Of the patients with ECE reported on MRI, $10.5 \%$ went on to have positive surgical margins compared to $41.4 \%$ of those patients without ECE on MRI $(\mathrm{p}=0.404)$.

Conclusion: At our centre, the use of preoperative 3T pelvic phase-array MRI in predicting pathological ECE is of questionable benefit. Our findings suggest that preoperative MRI reports of organ-confined disease may lead to closer surgical dissection and subsequent positive surgical margins, regardless of true pathological staging. As such, caution should be exercised when basing intraoperative decisions on MRI findings.

Source of Funding: This project is not funded.

Previously Presented: McMaster Resident Research Day

\section{Accuracy and clinical utility of percutaneous renal core biopsies and fine needle aspirations}

Premal Patel, Kamaljot Singh Kaler, lain Kirkpatrick, Tom McGregor

Department of Urology, University of Manitoba, Winnipeg, MB

Objective: The increasing incidence of renal cell carcinoma can largely be attributed to the detection of small renal masses on abdominal imaging. These masses are found incidentally and are often clinically asymptomatic. Numerous studies have demonstrated the effectiveness and safety of percutaneous renal biopsies in the management of these patients; however, this tool has failed to gain widespread use. Our study aims to assess the accuracy of percutaneous biopsies and how they have shaped the management of our patients.

Methods: A retrospective chart review was undertaken for patients who had a percutaneous renal biopsy at the St. Boniface General Hospital from October 2012 to February 2014. Patient age, gender, core biopsy and fine needle aspiration (FNA) results, procedure complications, and if applicable, final surgical specimen pathology was extracted from patient charts. Results: A total of 31 patients were found to have undergone percutaneous renal biopsies over the past year. The mean age was 68.4 years old (range 49-89). 29 patients had core biopsies whereas 31 had FNA. Of the 29 core biopsies, 15 (52\%) were found to be malignant, $6(21 \%)$ were benign, 5 $(17 \%)$ were non-diagnostic and $3(10 \%)$ of which malignancy could not be excluded. Of the FNA, $14(45 \%)$ were non-diagnostic, $8(26 \%)$ were malignant, $3(10 \%)$ were benign, $2(9.5 \%)$ were suspicious for malignancy and $2(9.5 \%)$ were found to have atypical cells. 7 patients underwent partial or radical nephrectomies during this period with core biopsy demonstrating final specimen pathology in 5 of the 7 patients $(71 \%)$ and FNA demonstrating similar pathology in 3 of the 7 samples (43\%).

Conclusion: With literature and our experience demonstrating minimal to no complications with percutaneous renal biopsies; it is an invaluable tool in the management of renal masses. Our results also question the utility of fine needle aspirations as they failed to provide any additional information to the core biopsy.

Source of Funding: This project is not funded. 